\title{
Article
}

\section{Investigations on Roller-Based Filament Drives}

\author{
Vinzenz Nienhaus, Dieter Spiehl (D) and Edgar Dörsam * (D) \\ Institute of Printing Science and Technology, Technical University of Darmstadt, Magdalenenstr. 2, \\ 64289 Darmstadt, Germany; nienhaus@idd.tu-darmstadt.de (V.N.); spiehl@idd.tu-darmstadt.de (D.S.) \\ * Correspondence: doersam@idd.tu-darmstadt.de; Tel.: +49-6151-1622780
}

Citation: Nienhaus, V.; Spiehl, D.; Dörsam, E. Investigations on Roller-Based Filament Drives. J. Manuf. Mater. Process. 2021, 5, 53. https://doi.org/10.3390/ jmmp5020053

Academic Editor: Steven Y. Liang

Received: 17 April 2021

Accepted: 18 May 2021

Published: 21 May 2021

Publisher's Note: MDPI stays neutral with regard to jurisdictional claims in published maps and institutional affiliations.

Copyright: (c) 2021 by the authors. Licensee MDPI, Basel, Switzerland. This article is an open access article distributed under the terms and conditions of the Creative Commons Attribution (CC BY) license (https:// creativecommons.org/licenses/by/ $4.0 /)$.

\begin{abstract}
The filament drive is a key part of the extrusion assembly of a Fused Filament Fabrication printer. This investigation examines the maximum feed force and the slip of different driving rollers using a filament made of polylactic-acid (PLA) on a test stand. The test stand systematically varies the main feed process parameters: feed velocity, pinch force between the rollers, and feed force. The maximum feed force has a characteristic dependency on the pinch force combined with a feedvelocity-dependency, which is influenced by the outer diameter of the driving roller. The slip was found to increase linearly with the feed force. The slip decreases with increasing pinch force and is nearly constant for pinch forces above $77 \mathrm{~N}-172 \mathrm{~N}$, depending on the driving roller tooth geometry and outer diameter. A model derived from contact mechanics was used for phenomenological modeling of the slip in relation to pinch force and feed velocity. An exponential ansatz provided good modeling of the slip at a constant pinch force. The model of the slip combined with the extrusion forces in the liquefier can be used to estimate the material flow in the future, thus leading to increased precision of the parts in a magnitude of systems.
\end{abstract}

Keywords: fused filament fabrication; fused deposition modeling; filament; filament drive; extruder; PLA

\section{Introduction}

A few percent of material flow deviation can lead to severe failure in demanding applications, like medical implants. A common 3d-printing method for customized medical implants is filament extrusion, commonly known as Fused Filament Fabrication (FFF) or under the brand name Fused Deposition Modeling-FDM [1]. It offers the opportunity of manufacturing functional parts out of a huge variety of polymers at very low investment costs. The low investment costs are related to the simple system design. The system is comprised of a material storage for the thermoplastic wire (filament), a feed mechanism (filament drive), and a liquefier in combination with a positioning system. The liquefier is responsible for melting and extrusion of the filament in the form of plastic strands with a smaller diameter than the filament. The strands make up a part, layer by layer.

The filament drive is typically called the extruder. It is also referred to as the cold end in contrast to the hot end, which includes the liquefier [2]. The filament drive has a huge influence on part quality. It is the only moving part in the extrusion system, comprised of a filament drive and liquefier, and is used to control the flow of plastic out of the liquefier. The filament drive typically features a set of rollers advancing the filament by friction and in the case of a toothed driving roller partly by form closure. Ideally, the system has a direct relationship between the angular velocity of the driving roller and the feed velocity of the filament. The real system has a slip, a percentual discrepancy between the angular and feed velocity, and is influenced by various other parameters. A good understanding of the filament drive is necessary, since precise control of the flow of plastic is a key parameter in promoting its industrial use in, e.g., the medical sector. 


\section{Evaluation of the State of the Art}

The filament drive has the task of converting electric energy and velocity information into a filament movement. The movement is accompanied by a feed force, which is the reaction of the process forces in the liquefier. The feed force is necessary to overcome the friction and flow forces in the liquefier and especially in the nozzle [3-7]. The higher the maximum applicable forces on the filament, the higher is the potential printing velocity [5].

Roller filament drives feature at least one toothed or rubbery roller (driving roller) and a counter-rotating part [8]. The filament is fed through these two axially parallel cylinders. The counter-rotating part can be another driving roller or an idler (pinch roller), which creates a normal force between the rollers (pinch force). Most printers feature a single-toothed driving roller in combination with an idling bearing. The advantages lie in the simple design of the filament drive, low cost and the simple theoretical relation between the driving motor's rotation and the filament's advance. There exist variations with two toothed and driven rollers [9] and additional sets of driving pairs. Plain driving rollers only rely on friction. Driving forces are limited by the friction coefficient and tolerable slip. Teeth are used to permanently deform the filament—creating a rack and pinion style gearing. The lack of understanding of the slip and maximum feed force of the filament drive within the magnitude of current printing systems leads to a lot of individual tuning for every printed part and printer.

There have been previous investigations on certain types of filament drives. The first investigation on comparable mechanisms can be found in wire arc welding. Neubert examined metal wire drives and found a dependency of the pinch force between the rollers and the feed force [10]. More in depth investigation on roller geometries and a universal test stand for feeding of welding wire was described by Reisgen [11]. Bellini stated existing "Navier-slip" in the filament drive, but did not carry out experiments specifically on the filament drive [3]. The motor torque and its limits was investigated by Lyu et al. [12]. Jiang and $\mathrm{Gu}$ investigated the process force-dependent slip of the feed velocity of an unspecified system [13]. They pulled a filament with a weight applied to it and measured the feed velocity. The simulated process force by weight application results in a slip of around $5 \%$ to $15 \%$. Mackay et al. measured the maximum feed force by "attaching a filament onto a force scale" [6]. They measured forces of $131 \pm 1 \mathrm{~N}$ for an acrylonitrile-butadiene-styrene (ABS) and $161 \pm 2 \mathrm{~N}$ for a polylactic-acid (PLA) filament. The maximum feed force of the filament drive in relation to the feed velocity was investigated by Go et al. [5]. They pushed the filament into the liquefier assembly and measured the applied force of the filament drive. A filament drive of a disassembled PrintrBot Metal was used [14]. They found an exponential decline of the maximum feed force in relation to the feed velocity. The forces ranged from $62 \mathrm{~N}$ at $0.08 \mathrm{~mm} / \mathrm{s}$ down to $42 \mathrm{~N}$ at $10 \mathrm{~mm} / \mathrm{s}$ for a type of ABS-filament. They also provided a model for failure of the filament drive based on the maximum shear force which provides an estimation of the maximum feed force within the magnitude of the measured values. Greeff and Schilling developed a closed-loop control of slip for the filament drive of a Renkforce RF1000 printer [15]. They measured a feed force and feed velocity-dependent slip of up to $80 \%$. The influence of the roller geometry on the maximum feed force has been investigated by Heywood in a blog entry [16]. Four different roller designs were tested in a simple measurement setup, but the maximum feed force was partly limited by the motor torque due to the varying diameters of the driving rollers. Prior investigation at the Institute of Printing Science and Technology in the form of two theses by Rohrmann and Schmitz provided the first data on the pinch-force-dependency [17] and materialdependency [18]. This investigation repeated core ideas, materials and methods of [19] with a new concept for evaluation of the maximum feed force and minimal pinch force.

Based on the previous findings, this current investigation takes a closer look at different roller geometries and their maximum feed force and slip in relation to the feed force and velocity. 
There are various influencing parameters on the filament drive resulting from the printing process, the filament drive itself, the filament, and the environment (given in Table 1).

Table 1. Influence parameters sorted by category and evaluated by their potential influence on the performance of the roller filament drive and the experiments. The source and explanation of the influence is given in the last column.

\begin{tabular}{|c|c|c|c|}
\hline Category & Influence Parameter & Influence & Sources/Explanation \\
\hline \multirow{5}{*}{ printing process } & pinch force & ++ & [17] \\
\hline & feed velocity & + & [13] \\
\hline & motor maximum torque & + & {$[12,16]$} \\
\hline & unwind force & - & minor systematic error \\
\hline & feed force & ++ & $\begin{array}{l}\text { max. force }[5,6] \\
\text { slip: }[10]\end{array}$ \\
\hline \multirow{5}{*}{ Filament drive } & roller geometry & ++ & {$[16,20]$} \\
\hline & & & big roller creates zone \\
\hline & idler geometry & - & $\begin{array}{l}\text { of nearly constant } \\
\text { contact pressure }\end{array}$ \\
\hline & number driven rollers & + & {$[9]$} \\
\hline & filament guidance & - & [11] \\
\hline \multirow{3}{*}{ Filament } & mechanical properties & + & [18] \\
\hline & diameter & + & $\begin{array}{l}\text { affects shear in [14], } \\
\text { input parameter of } \\
\text { Euler buckling [21] }\end{array}$ \\
\hline & $\begin{array}{l}\text { roundness/diameter } \\
\text { tolerance }\end{array}$ & - & minor systematic error \\
\hline \multirow{4}{*}{ Environment } & temperature & - & affects mechanical \\
\hline & humidits & & $\begin{array}{l}\text { properties of fllament } \\
\text { affects mechanical }\end{array}$ \\
\hline & humidity & - & properties of filament \\
\hline & dust/particles & - & $\begin{array}{l}\text { affects roller geometry, } \\
\text { cleaning necessary [22] }\end{array}$ \\
\hline
\end{tabular}

The relevant printing process influence parameters are pinch force, feed velocity, maximum motor torque, the unwind force (unwinding of rolled filament), and the feed force. The filament drive characteristic is also influenced by the filament drive's configuration, e.g., the geometry of the driving and idling roller, the number of driven rollers, and the filament guidance. The filament has an influence in the form of varying mechanical properties, the diameter, and the tolerance of roundness and diameter. The key environmental parameters are temperature, humidity, and dust, as well as particles. The evaluation of the influence parameters provides a basis for the development of a test stand especially suited to examine pinch force, feed velocity, feed force, and roller geometry as the main influence parameters. The filament's diameter, mechanical properties, and number of driven rollers will be covered in future research.

This study discusses state-of-the-art roller filament drives, which are used in most systems. The focus lies on the influence parameters: pinch force, feed velocity, and roller geometry. The resulting maximum feed force and slip is recorded with the aim to provide empirical insights into the workings of roller-based filament drives and to provide a model for the slip in a filament drive. This new approach provides a printer-independent view on the roller-based filament drive and the results are applicable to a wide range of printers. This enables the user to optimize their particular system.

\section{Materials and Methods}

Environmental parameters were kept constant at a temperature of $23^{\circ} \mathrm{C}$ and humidity of $50 \%$. The filament was provided by Prusa Research [23] and is made of clear Ingeo 
Biopolymer 4043D resin [24]. It has a nominal diameter of $1.75 \mathrm{~mm}$. Filament from two spools was used and measured five times with a micrometer (accuracy $0.001 \mathrm{~mm}$ ) at the beginning, middle, and end of the filament. The filaments on both filament spools have an average diameter of $1.752 \pm 0.003 \mathrm{~mm}$ and a roundness of $0.011 \pm 0.004 \mathrm{~mm}$.

Seven different driving rollers were used (see Table 2 and Figure 1). They differ mainly by the outer diameter and the tooth geometry. The tooth geometry for every driving roller was measured with optical microscopy or a caliper. The first three driving rollers are commonly used in Fused Filament Fabrication printers. The first driving roller is comparable to a straight toothed gear with flat flanks used in the printer CR-10 [25]. The second is used in Ultimaker 2+ printers [26] and is originally used for a $3 \mathrm{~mm}$ filament diameter. The third driving roller is named Hobb-Goblin [27] and is used in many printers derived from the Reprap project, like TAZ Workhorse Edition [28]. The first two driving rollers feature a varying knurling pattern with flat flanks, which is defined by the chordal pitch (width between two teeth) and the angle between the flanks of two teeth. Rollers \#5, \#6 and \#7 have a similar knurl type with a varying outer diameter. They are knurled standard parts which were modified to be mounted onto the driving stepper motor. The knurl type is referenced as described by DIN 82 [29]. The driving roller \#3 has machined grooves of elliptical shape and conforms slightly to the filament. The chordal pitch is $0.72 \mathrm{~mm}$ and the depth is $0.20 \mathrm{~mm}$. The plain roller (\#4) is a driving roller where the central plain region of a toothed roller is used (see Figure 1d). It is cylindrical with a diameter of $8.02 \mathrm{~mm}$ and a surface roughness $R_{z}$ of $10 \pm 5 \mu \mathrm{m}$. The roughness was measured at three different places with confocal microscopy using a PLu neox from Sensofar [30].

Table 2. Characteristic data of the driving rollers used in the experiments.

\begin{tabular}{ccccc}
\hline No & Description & Outer Diameter & Chordal Pitch & Angle \\
\hline$\# 1$ & Straight knurl (type RAA 10-105) & $10.90 \pm 0.01 \mathrm{~mm}$ & $1.00 \pm 0.01 \mathrm{~mm}$ & $90 \pm 1$ \\
$\# 2$ & Diamond knurl (type RGE 08-90) & $8.64 \pm 0.01 \mathrm{~mm}$ & $0.80 \pm 0.01 \mathrm{~mm}$ & $90 \pm 1$ \\
$\# 3$ & Hobb-Goblin, 35 teeth & $7.98 \pm 0.01 \mathrm{~mm}$ & $0.72 \pm 0.01 \mathrm{~mm}$ & elliptical \\
$\# 4$ & Plain roller & $8.02 \pm 0.01 \mathrm{~mm}$ & - & - \\
$\# 5$ & Straight knurl (type RAA 06-90) & $12.20 \pm 0.04 \mathrm{~mm}$ & $0.63 \pm 0.01 \mathrm{~mm}$ & $87 \pm 1$ \\
$\# 6$ & Straight knurl (type RAA 08-90) & $16.20 \pm 0.04 \mathrm{~mm}$ & $0.84 \pm 0.01 \mathrm{~mm}$ & $89 \pm 1$ \\
$\# 7$ & Straight knurl (type RAA 08-90) & $20.03 \pm 0.04 \mathrm{~mm}$ & $0.82 \pm 0.01 \mathrm{~mm}$ & $92 \pm 1$ \\
\hline
\end{tabular}

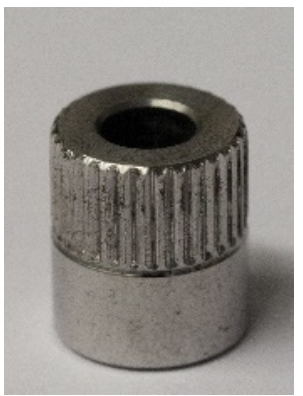

(a) \#1

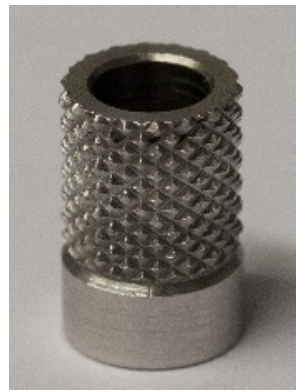

(b) $\# 2$

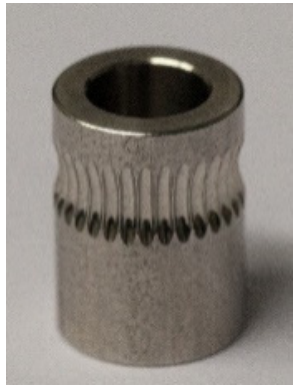

(c) \#3

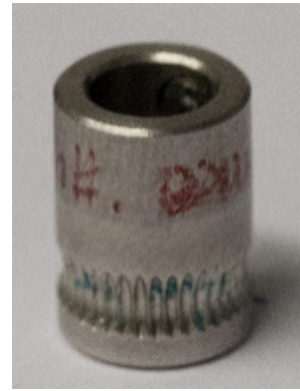

(d) \#4

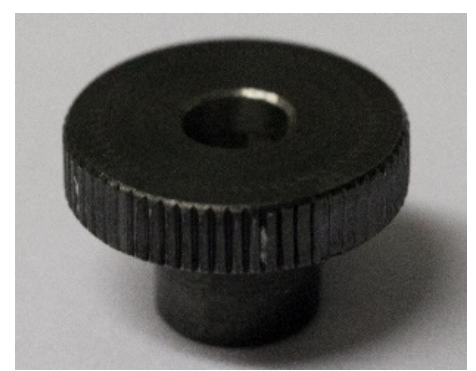

(e) $\# 5$

Figure 1. Images of the rollers (a)\#1, (b) \#2, (c) \#3, (d) \#4, and (e) \#6. Size and teeth data are given in Table 2.

The test stand features one driving motor with a counterpart for pinch force application and a friction-based process force simulator. Different setups in the form of a combination of driving motors and idlers are possible. A schematic free body diagram of the relevant components is given in Figure 2. The distance between the filament roll and the end of the process force simulator is around $60 \mathrm{~cm}$. This is comparable to a so-called Bowden-feedsystem, where there is a PTFE tubing between the liquefier and the filament drive. The filament is guided in PTFE tubes of $2 \mathrm{~mm}$ inner diameter between the encoder, the filament 
drive, and the process simulator. Near the filament drive, the tube is cut to the shape of the rollers and supports the filament as far as possible to prevent buckling.

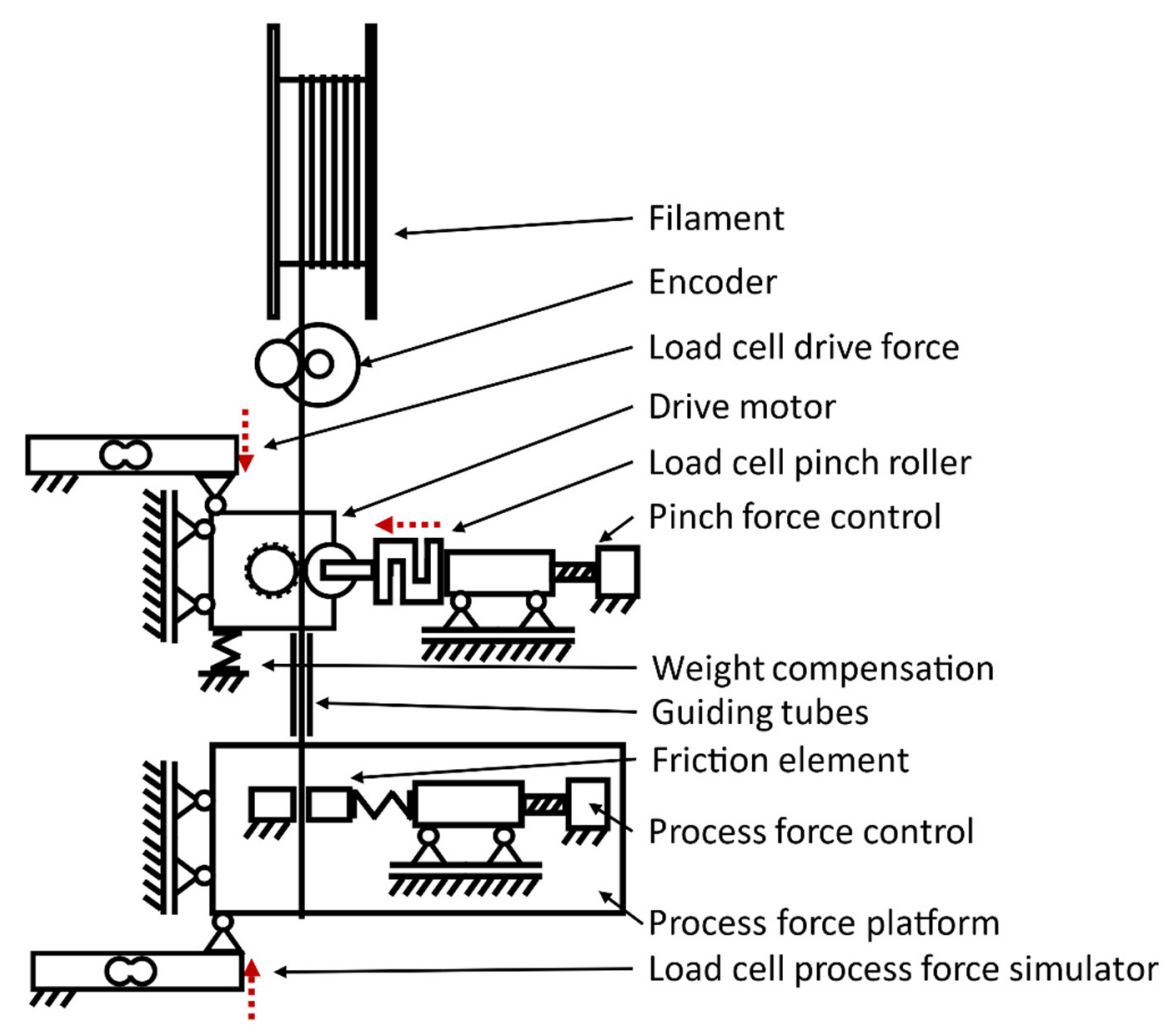

Figure 2. Free body diagram of the relevant components of the filament drive test stand derived from [19]. Measured forces are indicated as red dotted arrows.

The pinch force between the rollers is measured by a small form factor load cell rated $200 \mathrm{~N}$ of an unknown manufacturer. The load cell is directly attached to the mounting block of the idler. In the experiments a bearing of type 608 was used. The driving roller is turned by a 17HS3004-C5K geared stepper motor with a reduction gear ratio of 5.18 from Jugetek [31]. It provides a maximum torque of $1.35 \mathrm{Nm}$, which is sufficient for providing a maximum feed force of $135 \mathrm{~N}$ using the biggest $20 \mathrm{~mm}$ outer diameter driving roller. The motor is mounted onto a force measurement platform. It measures the feed force of the filament drive directly, eliminating any systematic errors of the guiding PTFE tubes. The pinch force is controlled by a motorized vise.

The process force simulator mimics the forces resulting from the liquefier. The process force is a result of the friction of the filament and the rheology of the melt and heavily depends on the liquefier temperature and feed velocity [7]. To provide stable process forces, it uses friction to create a coaxial force on the filament, which is being pushed by the filament drive. The process force simulator uses a motorized vise to clamp the filament in a v-grove with varying normal forces. It is mounted onto a load cell (H20A-C3-0020 from Bosche [32]) to measure the resulting friction forces. Forces up to a maximum of $200 \mathrm{~N}$ can be measured. All sensors are evaluated by a cRIO 9074 system from National Instruments [33]. The force measurement of all load cells was calibrated by weight application. The overall accuracy of the load cells was determined to be $\pm 2 \mathrm{~N}$ ( 2 sigma). The load cell measuring the driving force is influenced by the pinch force. It has an additional linearity error of $1 \%$ of the pinch force.

The filament is provided on a spool on top of the test stand. The filament advance is measured by an optical encoder that measures the feed velocity with a toothed roller. It has a measurement error of $0.05 \mathrm{~mm} / \mathrm{s}$ (2 sigma) for the range $0.1-15 \mathrm{~mm} / \mathrm{s}$. 
Every driving roller is characterized on the test stand with a set of process parameters. These are nominal feed velocity $v_{v i l, n o m}$ and nominal pinch force $F_{p, n o m}$. The percentual discrepancy between $v_{v i l}$ and $v_{f i l, n o m}$, calculated from the radial velocity from the angular velocity of the motor $\omega$ and the radius of the roller $r$, is defined as measured slip $s_{m}$ :

$$
s_{m}=\frac{v_{f i l, n o m}-v_{f i l}}{v_{f i l, n o m}}=\frac{\omega \cdot r-v_{\text {fil }}}{\omega \cdot r}
$$

The current feed velocity $v_{\text {fil }}$ is measured by the encoder. Note that the radius of the driving roller $r$ is subject to change and decreases with increasing pinch force. The error is part of $k_{s}$ in Equation (5) and $a, b$, and $c$ in Equation (8).

The nominal pinch force was varied from $10 \mathrm{~N}$ to $150 \mathrm{~N}$ in steps of $10 \mathrm{~N} ; 10 \mathrm{~N}$ to $20 \mathrm{~N}$ is the minimum pinch force for reliable feeding and $150 \mathrm{~N}$ is the point at which Euler-buckling (cf. [8]) in the $2 \mathrm{~mm}$ unsupported zone of the filament guide regularly occurs.

A common printing configuration is a filament diameter of $d_{i n}=1.75 \mathrm{~mm}$ and a nozzle capillary diameter $d_{\text {out }}=0.4 \mathrm{~mm}$. The relation between feed velocity $v_{\text {fil }}$ and printing velocity $v_{\text {printing }}$ derives from mass conservation. When a constant density is assumed, the relation is the following:

$$
v_{\text {fil }}=\frac{d_{\text {out }}{ }^{2}}{d_{\text {in }}^{2}} \cdot v_{\text {printing }}=\frac{0.4^{2}}{1.75^{2}} \cdot v_{\text {printing }} \approx 0.05 \cdot v_{\text {printing }}
$$

According to the relation, a feed velocity of $0.5 \mathrm{~mm} / \mathrm{s}$ corresponds to a relatively low printing velocity of $10 \mathrm{~mm} / \mathrm{s}$. The maximum feed velocity of $15 \mathrm{~mm} / \mathrm{s}$ mimics a very high printing velocity of $300 \mathrm{~mm} / \mathrm{s}$. The chosen set of feed velocities ranges from $0.5 \mathrm{~mm} / \mathrm{s}$ to $15 \mathrm{~mm} / \mathrm{s}$ in increasing steps $(0.5-1-2-5-10-15 \mathrm{~mm} / \mathrm{s})$. The parameters are changed in the following measurement algorithm given in pseudo-code here:

1. Set driving motor velocity to current $v_{f i l, n o m}$

2. Set and control pinch force to current $F_{p, n o m}$

3. Start measurement

4. While measurement is running

a. Increase simulated process force by $4 \mathrm{~N} / \mathrm{s}$

b. If $v_{f i l}<0.05 \mathrm{~mm} / \mathrm{s}$ (filament blockage measured by encoder)

i. Break

5. Decrease simulated process force to $0 \mathrm{~N}$ by $50 \mathrm{~N} / \mathrm{s}$

6. Wait $5 \mathrm{~s}$

7. Stop measurement

8. Jump to 1 using the next parameter set

The measurement is recorded continuously at a rate of 50 samples/s for every parameter set. Every parameter set is measured at least three times. This results in a minimum of 270 measurements per driving roller geometry, which are evaluated in the results.

The description of the slip in relation to the variable parameters feed velocity $v_{f i l}$, pinch force $F_{p}$ and feed force $F_{f}$ follows analytical descriptions of contact mechanics derived from Johnson [34]. The main ansatz for the slip $s$ is

$$
s=-\frac{4-3 v}{16 G r_{A}^{2}} F_{f}
$$

The slip $s$ is described in terms of the cross-contraction number $v$, shear modulus $G$, contact radius $r_{A}$, and feed force $F_{f}$. The relation is valid when slip takes place mainly in the contact region of an idealized plain roller and the filament due to the elastic deformation in the contact zone. The idea is to combine the material-dependent parameters to a parameter newly defined as the slip gradient $s^{\prime}$. Thus, further investigation on the parameter $s^{\prime}$ allows 
for separation of material parameters from the feed force. Furthermore, the slip gradient is a measure of the sensitivity to fluctuations of the process force. A filament drive with a low slip gradient is preferable.

The pinch force has an influence on the indentation depth and therefore, in this case idealized, on the contact radius $r_{A}$ (a measure of the contact surface in contact mechanics). We propose a linear relation between the pinch force $F_{p}$ and $r_{A}$ :

$$
r_{A} \sim F_{p}
$$

Equation (1) for the slip s reduces with the variable material-dependent parameter $k_{s}$ to

$$
s\left(F_{p}, F_{f}\right)=s^{\prime} \cdot F_{f}=\frac{k_{s}}{F_{p}^{2}} F_{f}
$$

From experiments [19] we know there is a constant offset $k_{p}$ and a constant slip gradient $k_{\infty}$. With the two parameters we derive the equation

$$
s\left(F_{p}, F_{f}\right)=s^{\prime} \cdot F_{f}=k_{p}+\left(\frac{k_{s}}{F_{p}^{2}}+k_{\infty}\right) \cdot F_{f}
$$

The slip $s$ is linearly dependent on feed force $F_{f}$ and reciprocally quadratic dependent on the pinch force $F_{p}$. The parameter $k_{S}$ is dependent on the material parameters. The filament's material exhibits viscoelastic properties. The time-dependency can be modeled with a material model based on the standard linear model in the Kelvin representation [34]. The model describes elastic behavior combined with an exponential decline of stresses to account for the viscous properties. Summarizing the material constants in three constants $a, b$, and $c$, it has the form

$$
\sigma(t)=\{a+b \cdot \exp \{(-c / t)\} \cdot \epsilon
$$

The stress $\sigma$ is related to the strain $\epsilon$ by a function with an exponential, time-dependent term and three constants. The constants $a$ and $b$ describe elastic properties, while $c$ contains elastic and viscous properties. The general response to different feed velocities $v_{f i l}$ is proposed to be characterized by this exponential decline (at a fixed range of $\left.F_{p}: k_{\infty} \hat{=} a, k_{s} / F_{p}^{2} \hat{=} b \cdot \exp \left(-c \cdot v_{f i l}\right)\right)$ :

$$
s\left(v_{f i l}, F_{f}, F_{p}=\text { const }\right)=s^{\prime} \cdot F_{f}=k_{p}+\left(a+b \cdot \exp \left(-c \cdot v_{f i l}\right)\right) \cdot F_{f}
$$

To sum up, we have Equation (5) for the pinch force relation of the slip $s$ and Equation (8) for a phenomenological description of the slip $s$ dependent on feed velocity $v_{f i l}$ and the test stand specific offset $k_{p}$.

\section{Results}

The recorded data of one measurement provide information about the feed force, feed velocity, and relevant control parameters of the test stand as raw data. In Figure 3 recorded data of the feed force measured by the feed force load cell and the feed velocity measured by the encoder are given.

The forces start to rise when the process force simulator increases the normal force in the friction element. After the feed force reaches a maximum, it suddenly decreases. The maximum feed force is stored for every measurement. The influence of pinch force, feed velocity, and driving roller geometry on the maximum feed force is discussed in Section 4.1. The feed velocity reaches a minimum after the point of maximum feed force. In the phase of load application, a decrease in velocity is visible. The measured feed velocity is dependent on the feed force. The measured slip is calculated by Equation (7) at every measurement index. Section 4.2 takes a closer look at the slip. The simulated process 
force is decreased rapidly after the failure of the filament drive and the filament moves again. There is a sudden increase in the feed velocity, due to the elastic energy stored in the filament while the process force builds up. This energy is suddenly released when the filament can move again. At high feed velocities the filament drive cannot resume filament movement. The driving roller eats into the filament because the friction element releases the process force too slowly. This creates a groove which the driving roller cannot escape. Especially high pinch forces promote this behavior. The filament is advanced manually in this case. A second failure mechanism is Euler-buckling. As described by Turner et al., the short unsupported sections between the driving roller and the guiding tube are susceptible to buckling [9]. At high pinch forces, the filament is rolled flat and buckling occurs regularly. In this case, the higher pinch forces need to be omitted. Both of the failure mechanisms are not investigated here because they are not relevant for the optimal parameter set based on the findings given in the next sections.



Figure 3. Sample of the recorded data of the feed force $F_{f}$ and feed velocity $v_{\text {fil }}$ measured at a pinch force of $110 \mathrm{~N}$ and $0.5 \mathrm{~mm} / \mathrm{s}$ nominal feed velocity $v_{f i l, \text { nom }}$ Maximum feed force $F_{f, \text { max }}$ and minimal feed velocity at blockage $v_{f i l, m i n}$ are marked with a star.

\subsection{Maximum Feed Force of the Filament Drive}

An evaluation of the maximum feed force of the driving roller \#1 (straight knurl) depending on the pinch force is given in Figure 4. The pinch force has a positive influence on the resulting maximum feed force in all measurements.

The maximum feed forces rise almost linearly and start to flatten at higher pinch forces. A second-order polynomial trend line provides a good fit for the total measurement set. There are maximum fluctuations of $25 \mathrm{~N}$ around the global trend line. The data are sorted by the different feed velocities. The bigger the surface area of the marker, the higher the velocity $(0.5-15 \mathrm{~mm} / \mathrm{s})$. There is a significant difference between the lowest and the highest feed velocities (see $F_{f, f i t, v_{f i l}=15.0}$ and $F_{f, f i t, v_{f i l}=0.5}$ in Figure 4 ). The maximum feed forces of lower feed velocities are up to $50 \mathrm{~N}$ higher at high pinch forces. The diamond knurl driving roller (\#2) exhibits a lower feed-velocity-dependency at higher pinch forces (see Figure 5). The trend line reaches $100 \mathrm{~N}$ at the highest pinch force, which is about $20 \mathrm{~N}$ lower than the trend line of roller \#1. Feed forces above $60 \mathrm{~N}$ are reached at $100 \mathrm{~N}$ pinch force, while the driving roller \#1 reaches $80 \mathrm{~N}$ at this point. 




Figure 4. Measurements of the maximum feed force in relation to the pinch force and feed velocity of the driving roller $\# 1-$ Straight knurl. The equation of the general 2 nd order polynomial fit is $F_{f, f i t}=-3.73 \cdot 10^{-3} F_{p}^{2}+1.27 F_{p}+6.52$.

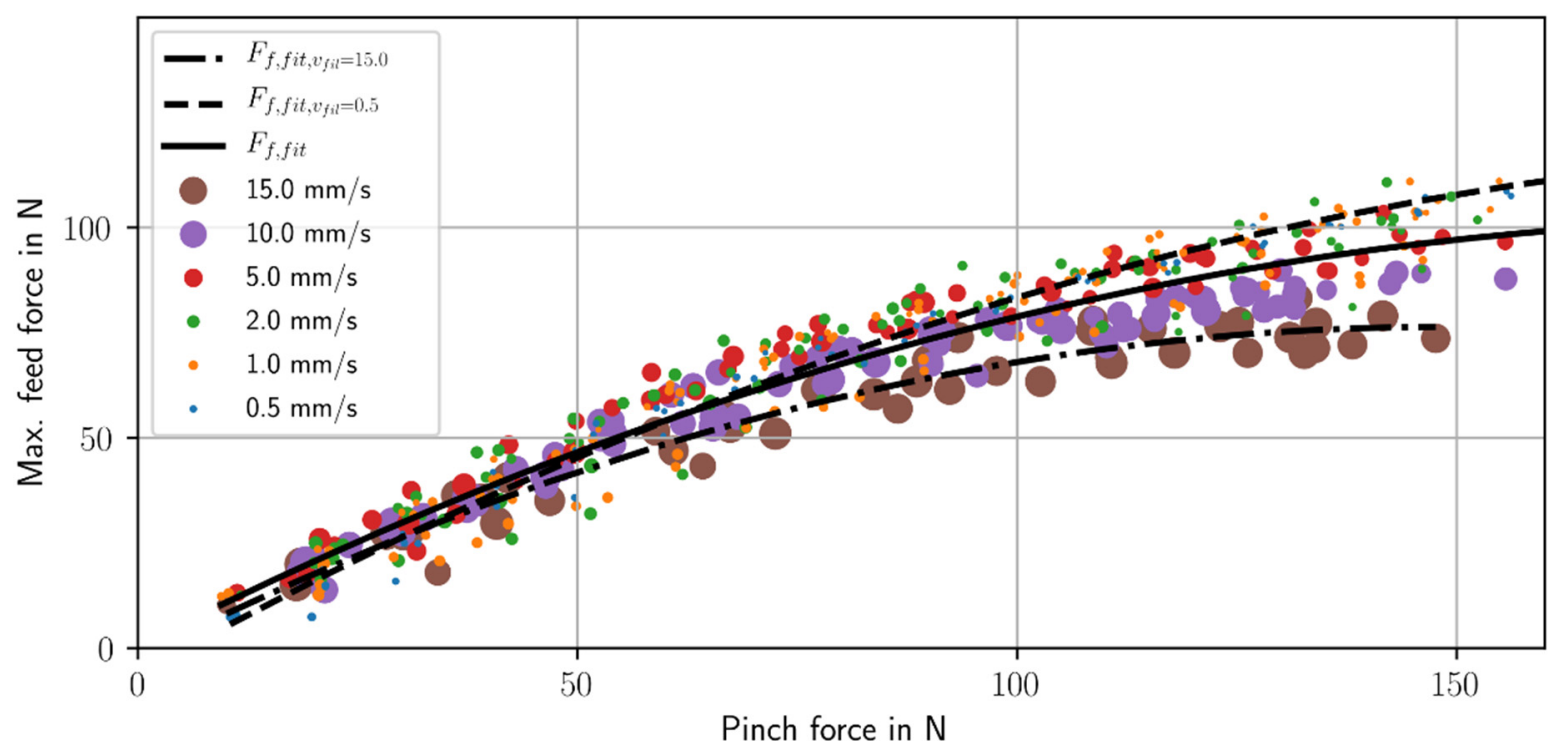

Figure 5. Measurements of the maximum feed force in relation to the pinch force and feed velocity for driving roller $\# 2-$ Diamond knurl. The equation of the general 2 nd order polynomial fit is $F_{f, f i t}=-2.78 \cdot 10^{-3} F_{p}^{2}+1.06 F_{p}+0.5$.

The maximum feed forces of the third driving roller Hobb-Goblin (\#3) in Figure 6 reach nearly the same level as the feed forces of the Diamond knurl driving roller (\#2) at the highest feed velocities. In contrast to \#1, there is no significant increase in the resulting maximum feed forces at these velocities and at a pinch force above $80 \mathrm{~N}$.

The plain roller without teeth (\#4) exhibits an inverted characteristic (see Figure 7). The lower the velocity, the lower are the achievable maximum feed forces. The overall shape of the data stays the same above a feed velocity of $0.5 \mathrm{~mm} / \mathrm{s}$ but at a lower average compared to the driving rollers \#1,\#2, and \#3. Above a pinch force of $150 \mathrm{~N}$ the idling bearing stops moving. The indentation force and friction in the bearing surpasses the tangential forces of the filament. 


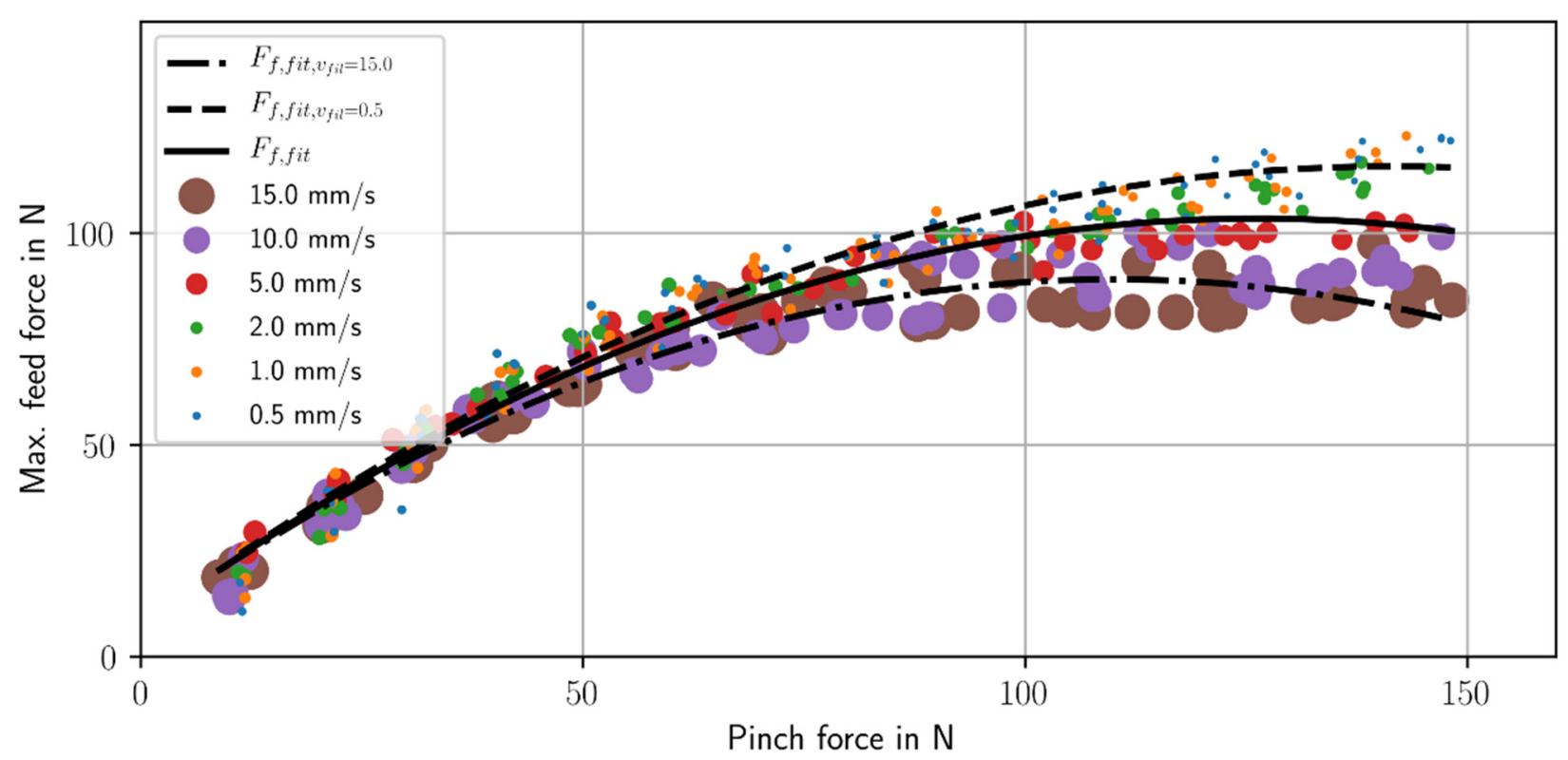

Figure 6. Measurements of the maximum feed force in relation to the pinch force and feed velocity for driving roller \#3-Hobb-Goblin. The equation of the general 2 nd order polynomial fit is $F_{f, f i t}=-6.03 \cdot 10^{-3} F_{p}^{2}+1.52 F_{p}+7.5$.

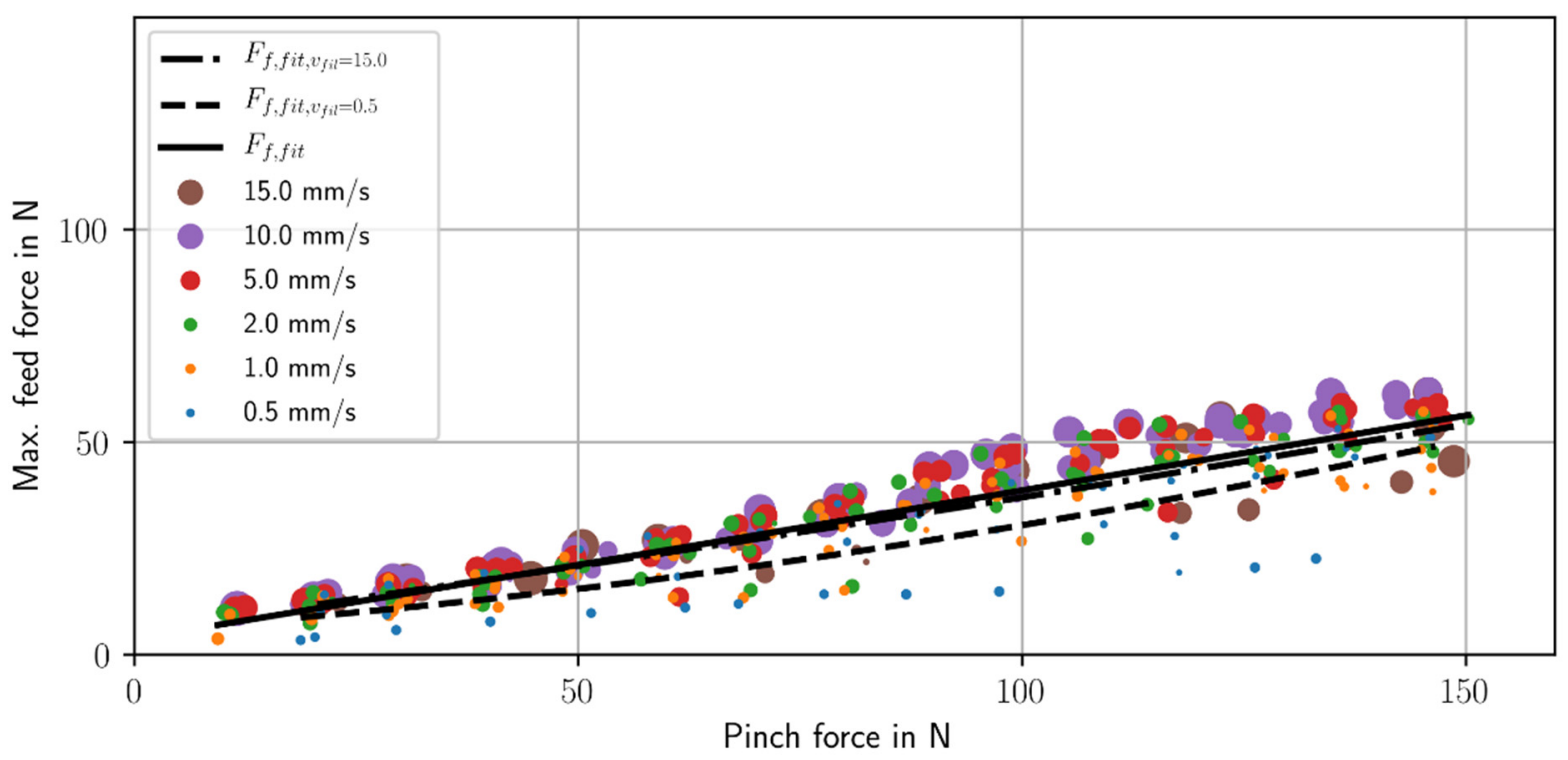

Figure 7. Measurements of the maximum feed force in relation to the pinch force and feed velocity for driving roller \#4-Plain roller. The equation of the general 2 nd order polynomial fit is $F_{f, f i t}=-2.80 \cdot 10^{-5} F_{p}^{2}+3.46 \cdot 10^{-1} F_{p}+3.7$.

The direct comparison of different outer diameters ranging from 12, 16, and $20 \mathrm{~mm}$ with comparable tooth geometry (rollers \#5,\#6, and 7) reveals a diameter influence on the feed velocity and maximum feed force relationship (Figure 8). The smallest roller (\#5) compares well to the feed velocity characteristic of the first roller with a straight knurl (see Figures 4 and 8 respectively). In the scatter diagram of roller \#6, with a diameter of $16 \mathrm{~mm}$, the points are mixed. They have no clear tendency of feed velocity influence. The driving roller with a diameter of $20 \mathrm{~mm}$ (\#7) shows an inverted influence of the feed velocity on the maximum feed force compared to the driving roller with a diameter of $12 \mathrm{~mm}$ (\#5). Higher velocities create higher maximum feed forces at a pinch force above $80 \mathrm{~N}$. 


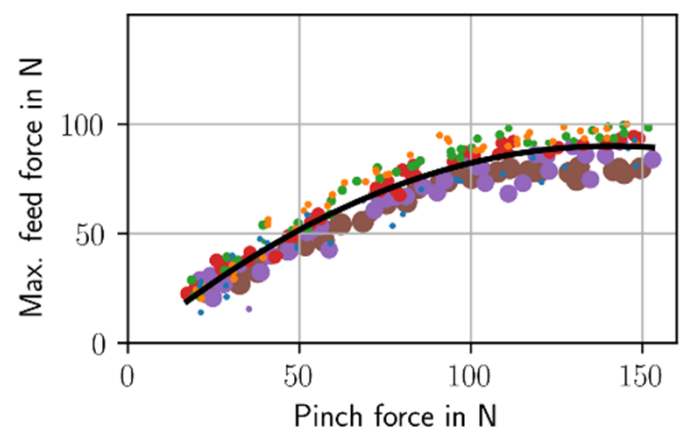

(a)

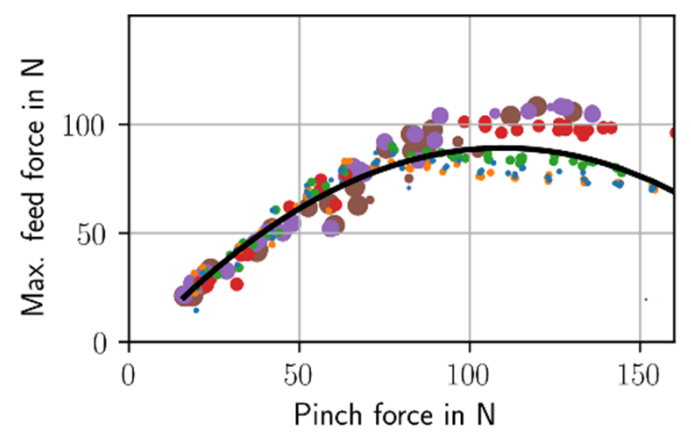

(c)

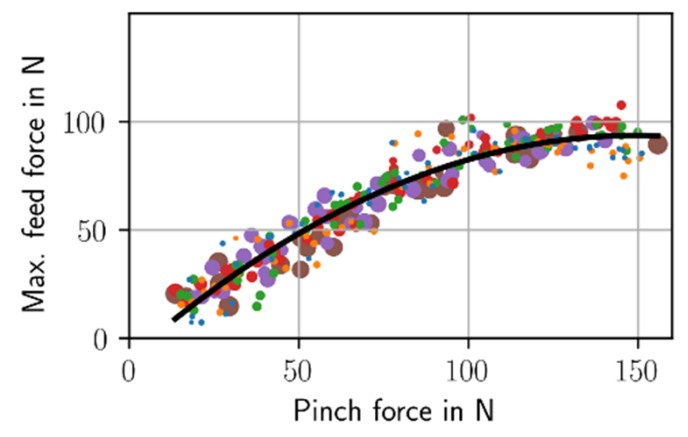

(b)

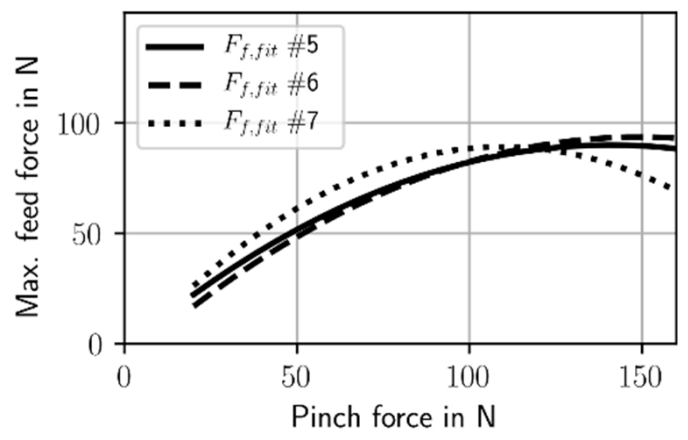

(d)

Figure 8. Measurements of the maximum feed force in relation to the pinch force and feed velocity of driving rollers (a) \#5 with an outer diameter of $12 \mathrm{~mm}-F_{f, f i t}=-4.64 \cdot 10^{-3} F_{p}^{2}+1.31 F_{p}-2.1,(\mathbf{b}) \# 6$ with an outer diameter of $16 \mathrm{~mm}-$ $F_{f, f i t}=-4.60 \cdot 10^{-3} F_{p}^{2}+1.37 F_{p}-8.7$, and (c) \#7 with an outer diameter of $20 \mathrm{~mm}-F_{f, f i t}=-7.86 \cdot 10^{-3} F_{p}^{2}+1.72 F_{p}-5.2$. A comparison of the trend lines is given in subfigure (d). Color and size of the data points are the same as in Figures $4-7$.

\subsection{Slip of the Filament Drive}

The main hypothesis is the dependency of slip $s$ on the feed force (see Equation (3)). In Figure 9a, a set of measured data of the slip of driving roller \#3 in relation to the feed forces at a feed velocity of $1 \mathrm{~mm} / \mathrm{s}$ and a pinch force of $80 \mathrm{~N}$ is given. An increase of the measured slip at higher feed forces is visible. Despite the fluctuations, a linear model is used. The fluctuations are caused by the indentation of the teeth, elastic compression of the filament and stick slip in the process force simulator. These dynamic effects are not further investigated here. The linear fit was applied to all measurements with good coefficients of determination (Figure 9b).

The dependency of the slip gradient on the pinch force for driving roller \#3 is given in Figure 10. The pinch force for every data point is given as the nominal setpoint. Small fluctuations around the setpoint during the measurement are neglected here. There is a decline of the slip gradient from $10 \mathrm{~N}$ to $60 \mathrm{~N}$ of pinch force and a nearly constant region above $60 \mathrm{~N}$. A minimum of the slip gradient is reached. It is not directly related to the indentation of the teeth, which fully penetrate at pinch forces around $100 \mathrm{~N}$. This was validated by samples of the indented filament. The penetration of the teeth and the cause of slip in the system are not discussed here in detail and will be subject to further investigation. The plain roller (\#4) does not exhibit a significant dependency of the slip on the pinch force (not shown, values given in Table 3). It has a constant relation between the pinch force and slip. The data were fitted with the quadratically reciprocal function in Equation (4), which provides a good fit of the data. The variable $k_{\infty}$ can be used as a measure for the minimal possible slip gradient. The gradient of driving roller \#3, for example, trends around $0.32 \% / \mathrm{N}$ in the region above $80 \mathrm{~N}$ pinch force. 


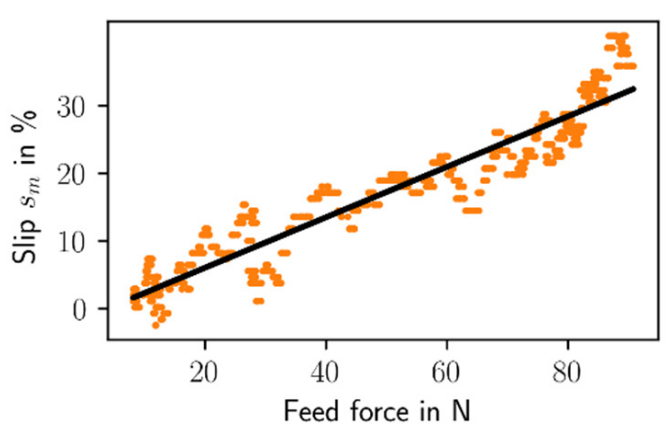

(a)

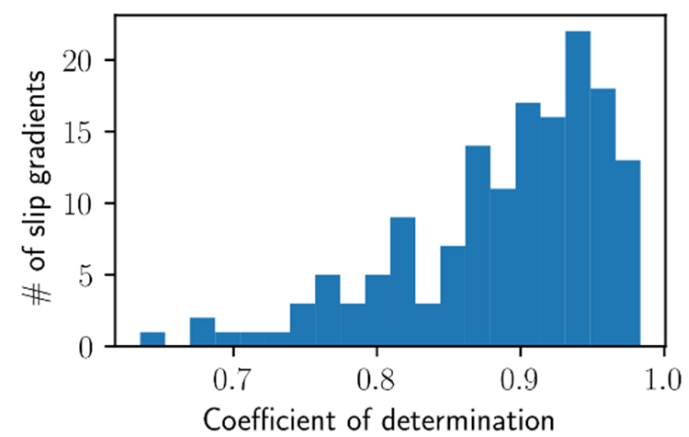

(b)

Figure 9. (a) A sample record of the measured slip $s_{m}$ (Equation (6)) in relation to the feed force at a feed velocity of $1 \mathrm{~mm} / \mathrm{s}$ and a pinch force of $80 \mathrm{~N}$ for driving roller \#3. A linear fit in form of a continuous black line is placed over the scattered orange data which represents the slip gradient $s^{\prime}$. (For the given setpoint: $s^{\prime}=0.33 \frac{\%}{N} \cdot F_{f}+0.63$ ). (b) Histogram of the coefficient of determination for all linear slip gradient regressions.

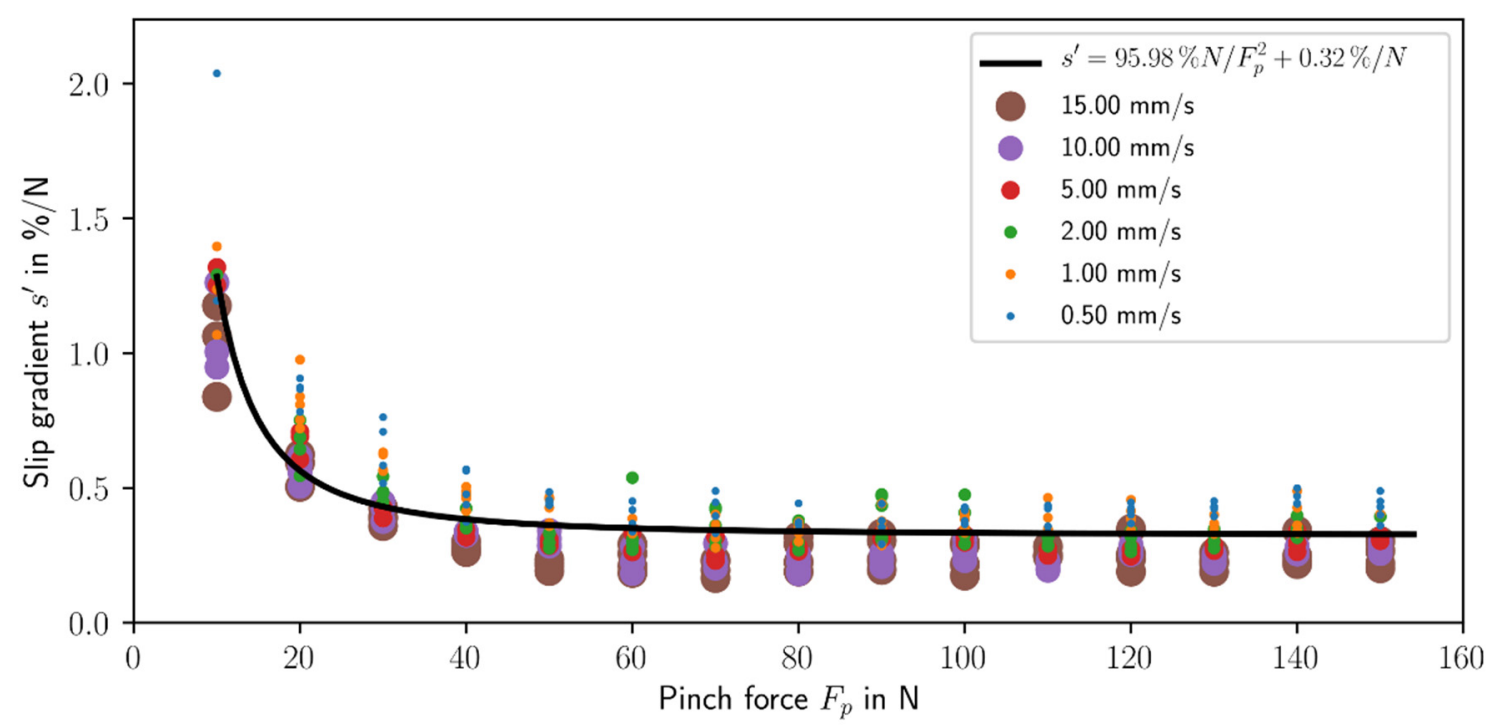

Figure 10. The slip gradient in relation to the pinch force for driving roller \#3 (Hobb-Goblin). The bigger the area of the data points the higher the feed velocity $(1 \mathrm{~mm} / \mathrm{s}$ to $15 \mathrm{~mm} / \mathrm{s})$. The fitted equation of $s^{\prime}$ of all data is overlaid as a continuous line.

Derived from Equation (6), we can deduce the minimal pinch force necessary for an acceptable minimal slip gradient $s^{\prime}$. The minimal pinch force $F_{p, \min }$ is where $s^{\prime}$ approaches $k_{\infty}$ by $10 \%$ :

$$
F_{p, \min }=\sqrt{\frac{k_{s}}{0.1 \cdot k_{\infty}}}
$$

The values of $F_{p, \min }$ are given in Table 3.

The deviation of the gradient in relation to the feed velocity is investigated at a constant range of pinch forces for all driving roller geometries. Figure 11 provides data about the average slip gradient and deviation for pinch forces from $80 \mathrm{~N}$ to $130 \mathrm{~N}$, which is well above the minimal pinch force of driving roller \#3, for example, and lower than the danger of buckling at the highest pinch forces. A decline of the gradient for higher feed velocities using driving roller \#3 is given. An exponential fit was used to model the data (see Equation (8)). Roller \#7, which has an outer diameter of $20 \mathrm{~mm}$, does not exhibit this behavior. It has no significant influence on feed velocity. Manufacturing tolerances of the driving roller cause higher fluctuations here. 



velocity parameters $a, b, c$ for all driving rollers.

\begin{tabular}{|c|c|c|c|c|c|c|}
\hline \multirow{2}{*}{$\begin{array}{l}\text { Driving } \\
\text { Rollers }\end{array}$} & \multirow{2}{*}{$F_{p, \min }$ in $\mathbf{N}$} & \multicolumn{2}{|c|}{$s^{\prime}\left(F_{p}\right)=\frac{k_{s}}{F_{p}^{2}}+k_{\infty}$} & \multicolumn{3}{|c|}{$s^{\prime}\left(v_{f i l}\right)=a+b \cdot \exp \left(-c v_{f i l}\right)$} \\
\hline & & $k_{s}$ in $\% \mathrm{~N}$ & $k_{\infty}$ in $\% / \mathrm{N}$ & $a$ & $b$ & $c$ \\
\hline$\# 1$ & 129 & $356.8 \pm 15.7$ & $0.22 \pm 0.01$ & $0.14 \pm 0.08$ & $0.16 \pm 0.07$ & $0.08 \pm 0.06$ \\
\hline$\# 2$ & 112 & $788.5 \pm 19.2$ & $0.63 \pm 0.02$ & $0.47 \pm 0.01$ & $0.37 \pm 0.02$ & $0.32 \pm 0.05$ \\
\hline \#3 & 77 & $96.0 \pm 3.0$ & $0.32 \pm 0.01$ & $0.24 \pm 0.01$ & $0.18 \pm 0.06$ & $0.32 \pm 0.01$ \\
\hline$\# 4$ & 44 & $404.8 \pm 76.3$ & $2.06 \pm 0.08$ & $1.24 \pm 0.12$ & $2.07 \pm 0.55$ & $0.73 \pm 0.29$ \\
\hline$\# 5$ & 100 & $286.8 \pm 18.3$ & $0.29 \pm 0.02$ & $0.23 \pm 0.01$ & $0.37 \pm 0.07$ & $0.83 \pm 0.18$ \\
\hline \#6 & 129 & $627.4 \pm 23.3$ & $0.38 \pm 0.01$ & $0.43 \pm 0.01$ & $\rightarrow \infty$ & $\rightarrow \infty$ \\
\hline \#7 & 172 & $643.0 \pm 34.2$ & $0.22 \pm 0.02$ & $0.29 \pm 0.01$ & $\rightarrow 0$ & $\rightarrow \infty$ \\
\hline
\end{tabular}

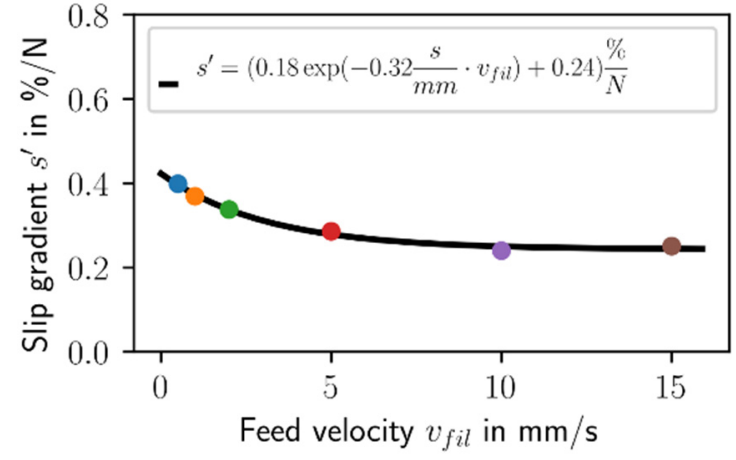

(a)



(b)

Figure 11. Dependency of the slip gradient on the feed velocity at pinch forces of $80 \mathrm{~N}$ to $130 \mathrm{~N}$ of driving roller (a) \#3-Hobb-Goblin and (b) \#7 with an outer diameter of $20 \mathrm{~mm}$. The fit function for $s^{\prime}$ is overlaid as a continuous line.

All driving rollers were analyzed in the same manner. The data for the minimal pinch force $F_{\mathrm{p}, \min }$, parameters of Equation (4) and parameters of Equation (6) are given in Table 3. Driving rollers \#6 and \#7 have no significant feed-velocity-dependency (see Figure 11b for \#7). The second term converges to zero in both cases because the negative exponent converges to infinity. The offset $a$ is a measure of the slip gradient at the highest feed velocities and is only valid for the pinch force range of $80 \mathrm{~N}-130 \mathrm{~N}$.

A practical estimation of the slip on a printer without feed-velocity-dependency at a certain pinch force $F_{p}$ can be calculated using Equation (6): The offset parameter $k_{p}$ has to be calibrated by measuring the mass flow in a given system at different setpoints and solving Equation (6) for $k_{p}$. A more precise prediction can be made by taking the feed-velocity-dependency into account using Equation (8). Note that variables $a, b$, and $c$ are valid for a pinch force range of $80 \mathrm{~N}$ to $130 \mathrm{~N}$ and variables a and b include material parameters as well as the factor $F_{p}^{-2}$ of Equation (6) at constant $F_{p}$. Under these assumptions, Equation (8) provides a direct relation of feed force and the resulting slip.

\section{Conclusions}

This investigation is the first in depth examination of the influence parameters of filament drives. For the characterization of filament drives, a universal test stand is described and for the examination of the influence parameters, extensive experimental data for a variety of driving rollers using PLA-filament are provided. The experimental data reveal a dependency of the maximum feed force on the pinch force and feed velocity, which was unknown for different driving roller designs. There is severe slip in the system, which can range to $30 \%$ just before failure of the filament drive. Thus, slip is a relevant quality issue in demanding applications. Depending on the process forces, a change of $2 \mathrm{~mm} / \mathrm{s}$ in feed velocity can lead to $2-3 \%$ slip, making stable printing processes with a controlled deposition of a desired material volume impossible. 
The maximum feed force of all the driving rollers increases with increasing pinch force. There exists a first region with a steep increase of the maximum force in relation to the pinch force and a second region with a reduced gradient. The relation is dependent on feed velocity. The guaranteed maximum feed forces reach $50 \mathrm{~N}$ to $80 \mathrm{~N}$ above pinch forces of $80 \mathrm{~N}$ for the toothed driving rollers (\#1-3,5-7). Toothed driving rollers with a diameter of less than $16 \mathrm{~mm}(\# 1, \# 2, \# 3$ and \#5) exhibit lower maximum feed forces at higher feed velocities. A system with a driving roller diameter of under $16 \mathrm{~mm}$ should be characterized at the highest reachable feed velocity since this results in the lowest maximum drive forces. The driving roller with a diameter of $20 \mathrm{~mm}$ exhibits an inverted feed-velocity-dependency and should be characterized at the lowest feed velocities for maximum feed force. The plain roller (\#4) is unsuitable for a filament drive because it exhibits unpredictable behavior at slow feed velocities and high overall slippage.

The part quality defining characteristic of slip in the filament drive is also dependent on the pinch force and feed velocity. A phenomenological model of the slip is developed and provides a prediction of slip, which is linearly dependent on the feed force. The slip gradient is a measure of the goodness of a driving roller and therefore the total filament drive. All driving rollers can be characterized by a minimal pinch force, at which the slip gradient reaches a minimum. Driving roller \#3 (Hobb-Goblin) has a low minimal pinch force of $77 \mathrm{~N}$ and a low slip gradient of $0.32 \% / \mathrm{N}$ which is the optimum of the relation, but the driving roller exhibits a dependency on feed velocity. Driving rollers \#6 and \#7 with outer diameters of $16 \mathrm{~mm}$ and $20 \mathrm{~mm}$ do not exhibit a significant relation of the slip on the feed velocity. This is a favorable behavior. A combination of the tooth design of driving roller \#3 with an increased diameter seems a good solution and should be investigated in future research. Since only a phenomenological approach for modeling is presented, more effort has to be put into the understanding of the indentation of the tooth in the filament. An exact solution of the contact problem with precise prediction of the slip is difficult due to the coupling of a three-dimensional contact problem with viscoelastic and plastic material properties and high deformations. Nevertheless, the presented model provides a good means for the practical use of slip prediction. A challenge for slip estimation and compensation arises from the unknown process forces in the liquefier, especially in the dynamic case. One proposed method is the integration of a force sensor in the liquefier system, as previously presented in the literature [4]. Another method is highly complex analytical modeling or simplified empirical modeling [7]. A model or empirical data can be used to predict the necessary feed force and the resulting slip in the filament drive and enhance material flow accuracy without adding additional error-prone sensors [19].

\section{Patents}

The slip compensation by modeling of the slip in the filament drive and mapping it to the measured process forces of a liquefier is the subject of a patent application (reference number 102020115 203.5).

Supplementary Materials: Supplementary materials can be found at https://www.mdpi.com/ article/10.3390/jmmp5020053/s1.

Author Contributions: Conceptualization, Investigation, Writing: V.N.; Writing-review and editing, D.S.; Supervision, E.D. All authors have read and agreed to the published version of the manuscript.

Funding: D.S. would like to thank the Merck KGaA for financial support through the Merck Lab ${ }^{\circledR}$ TU Darmstadt. We acknowledge the support of the Deutsche Forschungsgemeinschaft (DFGGerman Research Foundation) and the Open Access Publishing Fund of the Technical University of Darmstadt.

Institutional Review Board Statement: Not applicable.

Informed Consent Statement: Not applicable.

Data Availability Statement: Data is provided as Supplementary Materials. 
Conflicts of Interest: The authors declare no conflict of interest.

\section{References}

1. What Is FDM 3D Printing? Available online: https://www.stratasys.com/fdm-technology (accessed on 5 February 2020).

2. RepRap. Available online: https://reprap.org/wiki/RepRap (accessed on 5 February 2020).

3. Bellini, A.; Güçeri, S.; Bertoldi, M. Liquefier dynamics in fused deposition. J. Manuf. Sci. Eng. 2004, 126, 237. [CrossRef]

4. Coogan, T.J.; Kaazmer, D.O. In-line rheological monitoring of fused deposition modeling. J. Rheol. 2019, 63, 141-155. [CrossRef]

5. Go, J.; Hart, A.J. Fast desktop-scale extrusion additive manufacturing. Add. Manuf. 2017, 18, 276-284. [CrossRef]

6. Mackay, M.E.; Swain, Z.R.; Banbury, C.R.; Phan, D.D.; Edwards, D.A. The performance of the hot end in a plasticating 3D printer. J. Rheol. 2017, 61, 229-236. [CrossRef]

7. Nienhaus, V.; Smith, K.; Spiehl, D.; Dörsam, E. Investigations on nozzle geometry in fused filament fabrication. Add. Manuf. 2019, 28, 711-718. [CrossRef]

8. Turner, B.N.; Strong, R.; Gold, S.A. A review of melt extrusion additive manufacturing processes: I. process design and modeling. Rap. Prototyp. J. 2014, 20, 192-204. [CrossRef]

9. Bondtech. Available online: www.bondtech.se (accessed on 5 February 2020).

10. Neubert, G.; Goethe, D. Diagnose von drahtvorschubsystemen für schweibroboter und -automaten bei einsatz von sensoren und prozessrechner. Schweisstechnik 1984, 34, 371-373.

11. Reisgen, U. Untersuchungen von Drahtvorschubsystemen und deren Komponenten zur Verbesserung der Drahtelektrodenförderung beim Metall-Schutzgasschweissen; Shaker: Aachen, Germany, 1995; ISBN 3826505913.

12. Lyu, J.; Shen, H.; Wang, R.; Rudolph, N. Feasible solutions for precise 3D printing: The simulation and proposal. In Proceedings of the 2017 5th International Conference on Mechatronics, Materials, Chemistry and Computer Engineering (ICMMCCE 2017), Chongqing, China, 24-25 July 2017; Atlantis Press: Paris, France, 2017. ISBN 978-94-6252-381-4.

13. Jiang, K.Y.; Gu, Y.H. Controlling parameters for polymer melting and extrusion in FDM. KEM 2004, 259-260, 667-671. [CrossRef]

14. Go, J.; Schiffres, S.N.; Stevens, A.G.; Hart, A.J. Rate limits of additive manufacturing by fused filament fabrication and guidelines for high-throughput system design. Add. Manuf. 2017, 16, 1-11. [CrossRef]

15. Greeff, G.P.; Schilling, M. Closed loop control of slippage during filament transport in molten material extrusion. Add. Manuf. 2017, 14, 31-38. [CrossRef]

16. Heywood, M. 3D Printer Extruder Filament Drive Gear Review \& Benchmark. Available online: https://airtripper.com/1676/3 d-printer-extruder-filament-drive-gear-review-benchmark/ (accessed on 5 February 2020).

17. Rohrmann, M. Systematische Untersuchung des Materialvorschubsystems bei der Schmelzschichtung. Bachelor's Thesis, Technical University of Darmstadt, Darmstadt, Germany, 2017.

18. Schmitz, M. Materialabhängigkeit von Kunststoffdrahtvorschubsystemen für die additive Schmelzextrusion. Master's Thesis, Technical University of Darmstadt, Darmstadt, Germany, 2018.

19. Nienhaus, V. Untersuchung und Modellierung von Kunststoffextrusionssystemen in der Fused Filament Fabrication. Ph.D. Thesis, Technical University of Darmstadt, Darmstadt, Germany, 2020.

20. Fiedler, M. Evaluating tension and tooth geometry to optimize grip on 3D printer filament. 3D Print. Addit. Manuf. 2015, 2, 85-88. [CrossRef]

21. Venkataraman, N.; Rangarajan, S.; Matthewson, M.J.; Harper, B.; Safari, A.; Danforth, S.C.; Wu, G.; Langrana, N.; Guceri, S.; Yardimci, A. Feedstock material property_Process relationships in fused deposition of ceramics (FDC). Rap. Prototyp. J. 2000, 6, 244-253. [CrossRef]

22. Diabase Engineering. Available online: https:// flexionextruder.com (accessed on 5 February 2020).

23. Prusa Research. Available online: www.prusa3d.com (accessed on 5 February 2020).

24. Nature Works. Available online: www.natureworksllc.com (accessed on 5 February 2020).

25. Creality 3D Technology. Available online: www.creality3d.com (accessed on 5 February 2020).

26. Ultimaker. Available online: www.ultimaker.com (accessed on 5 February 2020).

27. E3D Online. Available online: www.e3d-online.com (accessed on 5 February 2020).

28. Aleph Objects. Available online: www.alephobjects.com (accessed on 5 February 2020).

29. Deutsches Institut für Normung e. V. Rändel; Beuth Verlag GmbH: Berlin, Germany, 1973; DIN 82.

30. Sensofar Tech. Available online: www.sensofar.com (accessed on 5 February 2020).

31. Jugetek. Available online: www.jugetek.com (accessed on 5 February 2020).

32. Bosche. Available online: www.bosche.eu (accessed on 5 February 2020).

33. National Instruments. Available online: www.ni.com (accessed on 5 February 2020).

34. Johnson, K.L. Contact Mechanics, 9th ed.; Cambridge University Press: Cambridge, UK, 2003; ISBN 0521347963. 